\title{
Measurements with electroweak gauge bosons at $\mathrm{LHCb}$
}

\section{Tara SHEARS}

University of Liverpool

E-mail: tara.shearsecern.ch

We have measured the $\mathrm{W}$ and $\mathrm{Z}$ boson production cross-sections in proton-proton collisions at a centre-of-mass energy of $7 \mathrm{TeV}$, using datasets of up to approximately $1 \mathrm{fb}^{-1}$ collected by the $\mathrm{LHCb}$ experiment. All measurements are based on boson decays with at least one lepton in the final state, which has transverse momentum $p_{T}$ exceeding $20 \mathrm{GeV}$ and pseudorapidity $\eta$ within $2.0-4.5$. W bosons are reconstructed in decays to muons. $\mathrm{Z}$ bosons are reconstructed in decays to electron, muon or tau lepton pairs, where the reconstructed invariant mass $m_{Z}$ lies in the range $60 \leq m_{Z} \leq 120 \mathrm{GeV}$. In all cases results are consistent with next-to-next-to-leading order perturbative QCD predictions.

The European Physical Society Conference on High Energy Physics 18-24 July, 2013

Stockholm, Sweden

\footnotetext{
* Speaker.

$\dagger$ on behalf of the LHCb collaboration.
} 


\section{Introduction}

Measurements of $\mathrm{W}$ and $\mathrm{Z}^{1}$ production in proton-proton collisions with a centre-of-mass energy of $7 \mathrm{TeV}$, constitute an important test of the Standard Model. Depending on boson rapidity, the dominant uncertainty arises from the underlying parton distribution functions (PDFs). The LHCb experiment [1] is instrumented with full tracking, calorimetry and particle identification capability between pseudorapidities $(\eta$ ) of 2 to 5 . Consequently, measurements made by LHCb can test the Standard Model and provide experimental constraints on the PDFs, both in a pseudorapidity region common to ATLAS and CMS $(2<\eta<2.5)$ and uniquely with full tracking information in the forward region $(\eta>2.5)$. We present results for the total $\mathrm{W}$ and $\mathrm{Z}$ cross-section within the $\mathrm{LHCb}$ kinematic acceptance, where the final state leptons have transverse momentum $p_{T}>20 \mathrm{GeV}$, lie within pseudorapidities of 2 to 4.5 , and, for $Z$ production, posess a dilepton invariant mass between 60 and $120 \mathrm{GeV}$. In addition, differential cross-section measurements as a function of $\mathrm{Z}$ boson $\phi^{* 2}$ and rapidity, and $\mathrm{W}$ decay lepton pseudorapidity, are reported.

\section{Event Selection}

All candidates are triggered by requiring at least one electron or muon with $p_{T}>20 \mathrm{GeV}$.

$\mathbf{Z} \rightarrow \mu \mu$

$\mathrm{Z}$ candidates must consist of an oppositely charged muon pair, where both muons lie within $2<\eta<4.5$ and possess $p_{T}>20 \mathrm{GeV}$, and with a dimuon invariant mass lying within $60<M_{\mu \mu}<$ $120 \mathrm{GeV}$. Background contributions from semileptonic decay of heavy flavour and hadrons misidentified as muons are estimated directly from data. Electroweak backgrounds $(\mathrm{Z} \rightarrow \tau \tau$, WW and top pair production) are estimated from simulation. Approximately $1 \mathrm{fb}^{-1}$ of data have been analysed. About $52600 \mathrm{Z}$ candidates are selected with a purity exceeding 99.7\%. The dimuon invariant mass distribution is shown in figure 1 (left).

\section{$\mathbf{Z} \rightarrow \mathbf{e e}$}

Candidate $\mathrm{Z}$ bosons must possess an oppositely charged electron pair, where both electrons lie within $2<\eta<4.5$ and have $p_{T}>20 \mathrm{GeV}$, and a dielectron invariant mass above $40 \mathrm{GeV}$. Backgrounds from heavy flavor and hadron misidentification are small and estimated from data. Electroweak backgrounds ( $\mathrm{Z} \rightarrow \tau \tau$ and top pair production) are estimated from simulation. A dataset of $945 \mathrm{pb}^{-1}$ was used to perform the measurement. The invariant mass of the $21400 \mathrm{Z}$ candidates selected is shown in figure 1 (right). Note that as electron track information, rather than calorimeter information, is used to estimate the electron momentum, the mass resolution has been widened by unrecovered bremstrahlung losses. Nontheless, the selection purity is estimated to be $95 \%$.

\section{$\mathbf{Z} \rightarrow \tau \tau$}

Candidates are selected in the topology where one tau decays to an electron or muon which triggers the event and has $p_{T}>20 \mathrm{GeV}$, and the other decays to an electron, muon, or single hadron with $p_{T}>5 \mathrm{GeV}$. Leptons must lie within $2<\eta<4.5$; due to the calorimeter acceptance, hadrons must lie within a restricted region $2.25<\eta<3.75$. To suppress heavy flavour and misidentification

\footnotetext{
${ }^{1}$ In this paper, $\mathrm{Z}$ refers to both $\mathrm{Z}$ and $\gamma^{*}$ production around the $\mathrm{Z}$ pole. Note that units are quoted throughout with $\mathrm{c}=1$.

${ }^{2}$ The angle $\phi^{*}$ is defined as $\phi^{*}=\frac{\tan ((\pi-|\Delta \phi|) / 2}{\cosh (\Delta \eta / 2)}$.
} 
backgrounds, tau decay products must be isolated and back to back. To suppress WW, top pair and $\mathrm{Z}$ decays to electron or muon backgrounds, tau decay products must have lifetime and a $p_{T}$ imbalance. Backgrounds involving heavy flavour and misidentification are estimated from data, other electroweak backgrounds from simulation. A dataset of approximately $1 \mathrm{fb}^{-1}$ yields about 990 candidates, with a purity ranging from 60 to $70 \%$ depending on final state.
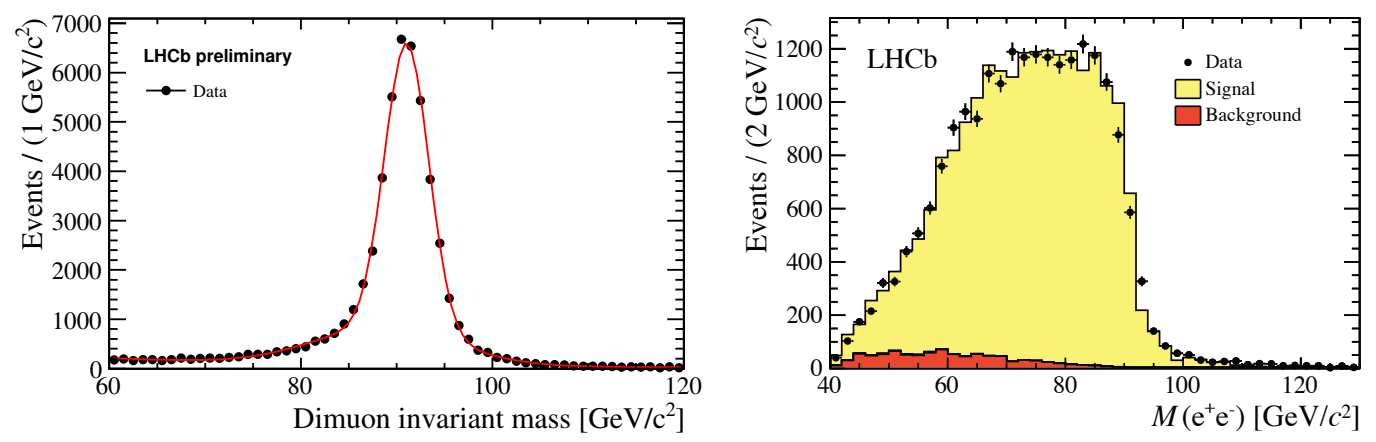

Figure 1: On the left, the invariant mass of $Z$ candidates in $\mu$ final states, shown as points and compared to a Crystal Barrel plus exponential fit. On the right, the invariant mass of $Z$ bosons in ee final states with templates of signal and background shapes overlaid.

$$
\mathbf{W} \rightarrow \mu v
$$

Candidate $\mathrm{W}$ bosons should possess one isolated, high transverse momentum $\left(p_{T}>20 \mathrm{GeV}\right)$ muon and little else. Muon candidates must lie within $2<\eta<4.5$, deposit little energy in the calorimetry to suppress pions and kaons punching through the calorimeter, and originate in the primary vertex to suppress heavy flavour. Events must not contain other muons to suppress muonic $\mathrm{Z}$ backgrounds. To evaluate the purity, the candidate muon $p_{T}$ spectrum is fitted to templates of the shapes expected for signal and electroweak backgrounds (obtained from simulation), and heavy flavour and misidentified hadron backgrounds (obtained from data). The normalisation of the $\mathrm{W}^{+}$ and $\mathrm{W}^{-}$signal, and the decay in flight background, are free parameters in the fit, which is shown in figure 2. Approximately 26000 candidates are selected from a dataset of $36 \mathrm{pb}^{-1}$, with a purity approaching $80 \%$.

\section{Cross-section measurement}

The cross-section $\sigma$ within a given rapidity, pseudorapidity, boson $p_{T}$ or $\phi^{*}$ interval is given by

$$
\sigma=\frac{\rho N f_{f s r}}{\varepsilon A L}
$$

where $\rho$ is the estimated purity, $N$ the number of candidates, $\varepsilon$ the candidate reconstruction efficiency, $A$ the acceptance and $L$ is the integrated luminosity of the dataset used to perform the measurement. As the cross-section is calculated at Born level, the final state radiation is accounted for by the correction $f_{f s r}$. Both $f_{f s r}$ and $A$ are determined from simulation.

The reconstruction efficiency contains components arising from the trigger, the tracking, the lepton or hadron identification, and the selection efficiency. Where possible, these are estimated 

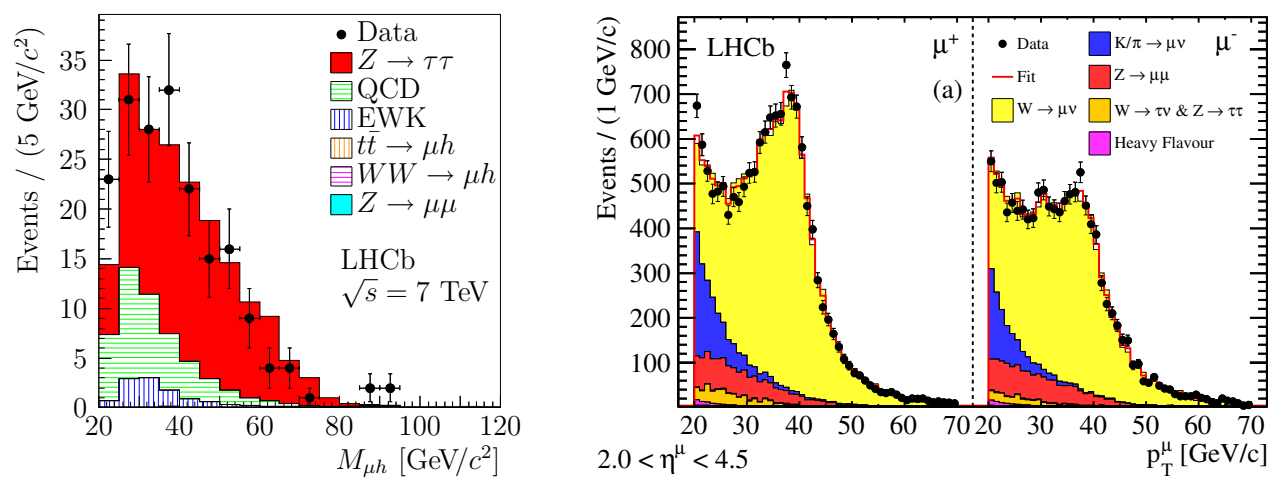

Figure 2: Invariant mass of $\mathrm{Z}$ candidates in $\tau$ final states (left), where the two $\tau$ leptons decay to a muon and a hadron. The muon transverse momentum spectrum from $\mathrm{W}$ decays, fitted to template shapes representative of signal and backgrounds to determine the sample purity (right).

from data using a tag-and-probe technique and the statistical precision of the determination used as the systematic uncertainty. Where quantities have been estimated from simulation, any difference between distributions in data and simulation is used to evaluate a systematic uncertainty.

\subsection{Systematic uncertainties}

Systematic uncertainties arise from: background estimation; the efficiency estimation; potential reconstruction biases; final state radiation corrections and acceptance; the integrated luminosity assumed. The uncertainties on backgrounds arise from Monte Carlo statistics and/or available statistics in data. The uncertainty in efficiency is taken as the statistical precision of the efficiency determination in data, and encompasses differences between data and simulation where calculated on Monte Carlo data. Potential charge-dependent reconstruction biases are estimated by comparing central values obtained with the dataset split into both magnet polarities. Acceptance and final state radiation uncertainties are estimated from simulation. The luminosity is determined by two methods, a Van Der Meer scan, and a beam gas method [2]. Both methods give similar results and are estimated to have a precision of order $3.5 \%$. This is the leading cause of systematic uncertainty for $\mathrm{W}$ and $\mathrm{Z}$ production in the muon and electron channels. For $\mathrm{Z}$ production of tau leptons, the efficiency uncertainty dominates. All sources are combined in quadrature to yield the final systematic uncertainty.

\section{Results}

\subsection{Z boson production}

Measurements of the inclusive $\mathrm{Z}$ cross-section (shown in table 1) are in agreement for all lepton final states, and in agreement with next-to-next-to-leading-order (NNLO) predictions.

Differential cross-sections have also been measured as a function of the $\mathrm{Z}$ boson rapidity and the $\phi^{*}$ angle, using muon and electron final states. Results for muon final states are given in these proceedings. The ratio of predicted to measured cross-section is shown as a function of $\mathrm{Z}$ rapidity in figure 3 (left). Ratios estimated at NNLO using FEWZ [6], next-to-next-to-leading-log 


\begin{tabular}{|l|c|}
\hline Process & Cross-section (pb) \\
\hline $\mathrm{Z} \rightarrow \mu \mu$ & $75.4 \pm 0.3 \pm 1.9 \pm 2.6[3]$ \\
$\mathrm{Z} \rightarrow$ ee & $76.0 \pm 0.8 \pm 2.0 \pm 2.6[4]$ \\
$\mathrm{Z} \rightarrow \tau \tau$ & $71.4 \pm 3.5 \pm 2.8 \pm 2.5[5]$ \\
$\mathrm{Z} \rightarrow \ell \ell$ & $74.7_{-1.4}^{+1.6} \pm 0.4[6]$ \\
\hline
\end{tabular}

Table 1: Inclusive $\mathrm{Z}$ production cross-sections, measured within the fiducial region defined previously. The first error quoted is statistical, the second systematic, and the third the luminosity uncertainty. The NNLO prediction given by FEWZ is listed in the last line of the table.

and approximated NNLO using ResBos [7], and at NLO (next-to-leading-order) using POWHEG interfaced to Pythia parton showering [8], are shown. All are in agreement. The FEWZ estimates include uncertainties due to PDFs and scale; ResBos uncertainties also include an estimate of non-perturbative effects, and only statistical uncertainties are considered for POWHEG estimates. The ratio of predicted to measured cross-section is shown as a function of $\phi^{*}$ in figure 3 (right). Here agreement with NNLO diverges at low values of $\phi^{*}$, but both ResBos and POWHEG show reasonable agreement throughout.
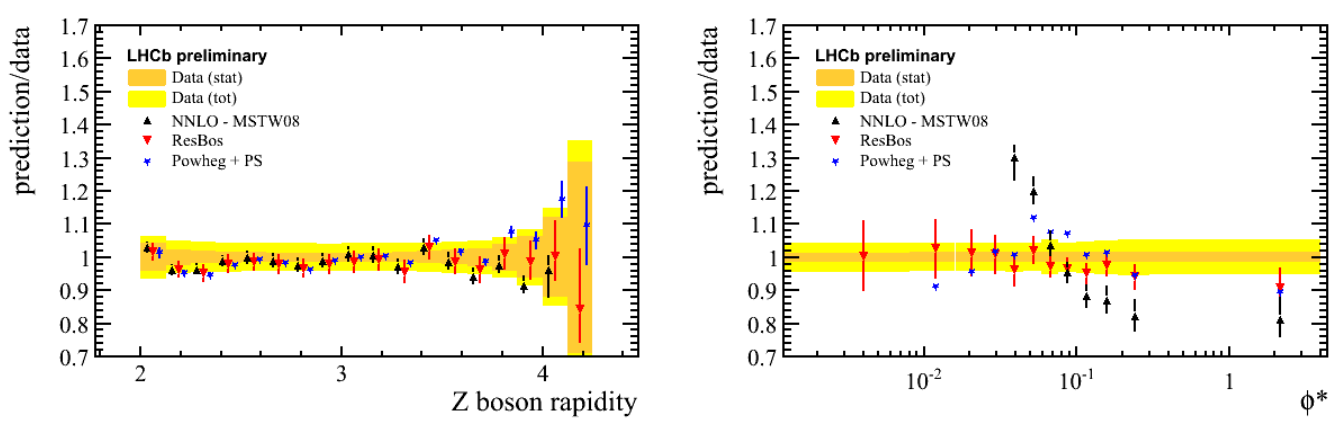

Figure 3: Ratio of predicted to measured differential cross-sections as a function of $\mathrm{Z}$ rapidity (left) and $\phi^{*}$ (right). The uncertainty in data is shown by the yellow band; the three theoretical predictions by the red, black and blue points.

\section{2 $\mathrm{W}$ boson production}

The $\mathrm{W}^{+}\left(\mathrm{W}^{-}\right)$boson cross-section, measured inside the fiducial region defined previously, is $831 \pm 9 \pm 27 \pm 29 \mathrm{pb}(656 \pm 8 \pm 19 \pm 23 \mathrm{pb})$, where the first, second and third uncertainties refer to statistical, systematic and luminosity uncertainties respectively. The measurements are consistent with NNLO predictions using a variety of PDF sets [9].

Differential cross-sections are measured as a function of muon pseudorapidity for $\mathrm{W}^{+}$and $\mathrm{W}^{-}$ production and shown in figure 4 (left). Here LHCb measurements (blue) have been extrapolated to the fiducial region of the published ATLAS measurements (green) to allow a direct comparison, following the prescription given in [10]. The W charge asymmetry (blue) is shown compared to the CMS measurement (red), for muons with transverse momenta exceeding $25 \mathrm{GeV}$, in figure 4 (right). Here both measurements use the same fiducial definition and can be compared directly. It can be seen that $\mathrm{LHCb}$ measurements are consistent with those of both experiments. 

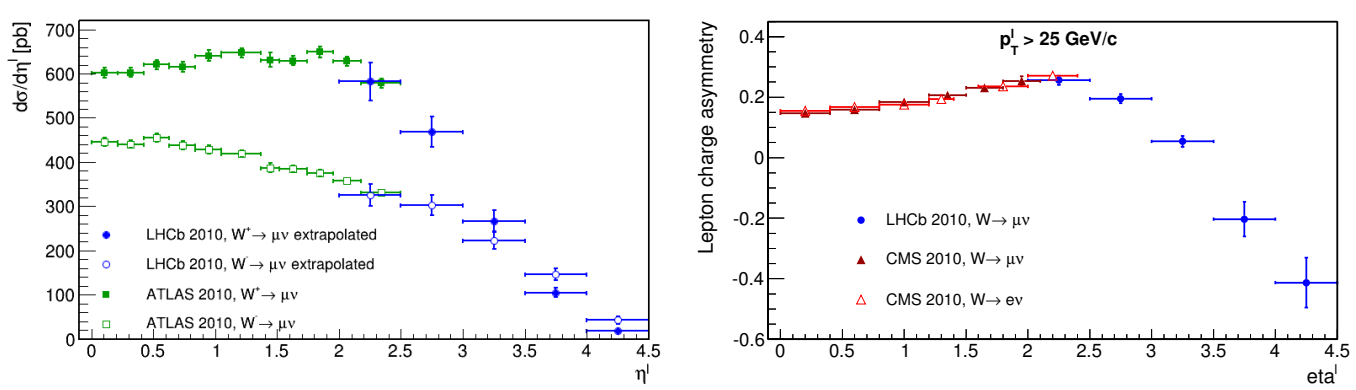

Figure 4: Differential $\mathrm{W}^{+}$and $\mathrm{W}^{-}$boson production, shown on the left, and $\mathrm{W}$ charge asymmetry, shown on the right, as a function of muon pseudorapidity. $\mathrm{LHCb}$ measurements are shown in blue, and are compared to those made by ATLAS and CMS as described in the text.

\section{Conclusions}

The $\mathrm{W}$ and $\mathrm{Z}$ boson production cross-sections have been measured in proton-proton collisions at a centre-of-mass energy of $7 \mathrm{TeV}$, using datasets of up to approximately $1 \mathrm{fb}^{-1}$ collected by the LHCb experiment. All measurements are based on boson decays with at least one lepton in the final state, which has transverse momentum exceeding $20 \mathrm{GeV}$ and pseudorapidity within $2.0-4.5$. W bosons are reconstructed in decays to muons. $\mathrm{Z}$ bosons are reconstructed in decays to electron, muon or tau lepton pairs, where the reconstructed invariant mass $m_{Z}$ satisfies $60 \leq m_{Z} \leq 120 \mathrm{GeV}$. Results are consistent with NNLO predictions, with the exception of the differential $Z$ production as a function of $\phi^{*}$ which is described better by ResBos and POWHEG. Measurements made by $\mathrm{LHCb}$ are in agreement with those obtained by ATLAS and CMS.

\section{References}

[1] A. Augusto Alves et al. JINST 3:S08005, 2008.

[2] S. Van Der Meer, ISR-PO/68-31, 1968; M. Ferro-Luzzi, CERN-PH-EP/2005-023 (2005).

[3] The LHCb collaboration, LHCb-CONF-2013-007

[4] The LHCb collaboration, JHEP02 (2013) 106.

[5] The LHCb collaboration, JHEP01 (2013) 111.

[6] R. Gavin, Y. Li, F. Petriello and S. Quackenbush, arXiv:1011.3540.

[7] G. A. Landinsky and C. P. Yuan, arXiv:hep-ph/9311341; C. Balazs and C. P. Yuan, arXiv:hep-ph/9704258; F. Laundry, R. Brock, P. M. Nadolsky and C. P. Yuan, arXiv:hep-ph/0212159.

[8] P. Nason, arXiv:hep-ph/0409146; S. Frixone, P. Nason and C. Oleari, arXiv:0709.2092; S. Alioli, P. Nason, C. Oleari and E. Re, arXiv:1002.2581.

[9] The LHCb collaboration, JHEP06 (2012) 058.

[10] The LHCb collaboration, LHCb-CONF-2013-005. 